\title{
Functional analysis of a novel parasitic nematode-specific protein of Setaria digitata larvae in Culex quinquefasciatus by siRNA mediated RNA interference
}

\author{
Magalla Bastian Chalitha Lakmal Somarathne', Yasanthi Illika Nilmini Silva Gunawardene², \\ Naduvilath Vishvanath Chandrasekharan ${ }^{1}$, Arjuna Nisantha Bandara Ellepola ${ }^{3}$ and Ranil Samantha Dassanayake ${ }^{1 *}$
}

\begin{abstract}
Background: Functional analysis of animal parasitic nematode genes is often quite challenging due to the unavailability of standardised in vitro culture conditions and lack of adequate tools to manipulate these genes. Therefore, this study was undertaken to investigate the suitability of Culex quinquefasciatus, as an in vivo culture platform for Setaria digitata larvae and RNA interference (RNAi), as a post-transcriptional gene silencing tool to study the roles of a vital gene that encodes a novel parasitic nematode-specific protein (SDNP).

Results: The red colour fluorescence detected following RNAi injection to the thorax of C. quinquefasciatus indicated the uptake of dsRNA by S. digitata larvae. The reduction of SDNP transcripts in siRNA treated larvae compared to nontreated larvae, as determined by qPCR, indicated that the siRNA pathway is operational in S. digitata larvae. The observation of motility reductions and deformities during the development indicated the association of SDNP in larvae locomotion and development processes, respectively. The irregularities in the migration of larvae in mosquitoes and elevated survival rates of mosquitoes compared to their untreated counterparts indicated reduced parasitism of $S$. digitata larvae in mosquitoes upon targeted downregulation of SDNP by siRNA treatment.

Conclusion: SDNP plays vital roles in muscle contraction, locomotion, development processes, larval development and parasitism of $\mathrm{S}$. digitata. Its ubiquitous presence in parasitic nematodes and its absence in their hosts provide a tantalising prospect of the possibility of targeting SDNP for future development of anthelmintic drugs. The susceptibility of the larval stages of $\mathrm{S}$. digitata for RNAi in Culex quinquefasciatus was also demonstrated for the first time in this study.
\end{abstract}

Keywords: Setaria digitata, SDNP, RNAi, Culex quinquefasciatus, Gene knockdown

\section{Background}

Nematodes are the most abundant metazoans, capable of inhabiting both terrestrial and aquatic habitats. This facilitates their ability to cause both human and animal health problems, as well as to impair crop production worldwide. Gastrointestinal nematodes infect 3.5 billion humans annually worldwide, and cause around 125,000 deaths per year, whereas filarial nematodes infect 120 million people in the globe annually, disabling around

\footnotetext{
* Correspondence: rsdassanayake@chem.cmb.ac.lk

${ }^{1}$ Department of Chemistry, Faculty of Science, University of Colombo,

Colombo 03 00300, Sri Lanka

Full list of author information is available at the end of the article
}

40 million of them [1-3]. Additionally, plant-parasitic nematodes destroy approximately $12.3 \%$ of the annual crop yield, and animal parasitic nematodes kill millions of livestock animals. This contributes to a loss of billions of US dollars annually and necessitates the allocation of additional billions of US dollars for anthelminthic drugs for livestock $[4,5]$. Strategies for controlling this problem have mainly focused on chemical treatments [5]. However, due to the limited efficiency and high toxicity of the available drugs, adverse effects on the environment, and emergence of drug resistance in parasites, these chemical treatment methods have become somewhat ineffective [6-8]. Hence, global interest has recently arisen in

(c) The Author(s). 2018 Open Access This article is distributed under the terms of the Creative Commons Attribution 4.0 International License (http://creativecommons.org/licenses/by/4.0/), which permits unrestricted use, distribution, and 
developing more effective controlling strategies for parasitic nematodes. In this context, screening of the nematode genome will enable us to gain a comprehensive knowledge of the molecular mechanisms associated with the development of the nematode, which may facilitate the control of parasitic nematodes $[9,10]$.

RNA mediated gene silencing has become a promising and unbiased tool in genetic screening [11]. RNA interference (RNAi) is a double-stranded RNA (dsRNA) induced post-transcriptional, homology-dependent gene silencing mechanism, naturally occurring in organisms, assumed to be involved in defence against virus attacks, heterochromatinisation and retrotransposon silencing [11-13]. RNAi was initially utilised in the genetic screening of Caenorhabditis elegans, and after that, this technique was expanded to other invertebrates (nematodes, insects, molluscs), vertebrates (fish, mice, humans), plants, fungi and algae [14-17]. RNAi allows the temporary shut-down of gene expression more specifically and rapidly and gives an insight into gene function $[18,19]$.

Setaria digitata is a parasitic nematode that naturally resides in the peritoneum and mesentery of cattle, zebu and water buffalo. Under normal circumstances, the nematode does not show any pathogenicity towards the natural hosts. The microfilariae produced by the adult female worm travel into the bloodstream of the cattle and are transmitted into new hosts via mosquito bites. However, when the larvae are transfected into non-permissive hosts such as goats, sheep and horses, they invade the brain, spinal cord, and eyes, causing cerebrospinal setariosis, conjunctivitis, and filariasis oculi, respectively [20]. Intriguingly, studies on S. digitata have revealed that it has a high resemblance to the human parasitic nematode Wuchereria bancrofti with regard to its morphology, antigenicity and histology [21, 22]. Hence $S$. digitata has been recommended as a model organism for studying $W$. bancrofti and Brugia malayi [23].

Unveiling the biology of the parasitic nematodes genes, which are specific and functionally crucial for their survival, is crucial in identifying targets to control the nematode population [16]. SDNP is such a gene found in $S$. digitata. Intriguingly, this gene has demonstrated high sequence similarities with putative proteins of $W$. bancrofti (79\%), Brugia malayi (77\%) and Loa loa (81\%). Moreover, bioinformatics studies of SDNP revealed that this protein contains a nuclear localisation signal, an RNAP_Rpb7_N_like domain, and an inter-domain linker of muscle-specific twitchin kinase similar to that of $C$. elegans [24]. Considering the background, our study focused on the functional characterisation of SDNP.

Commonly, RNAi protocols are practised by isolation of the nematodes from their natural hosts and maintaining them in artificial liquid cultures (in vitro). These in vitro protocols reduce the efficiency of RNAi due to several shortcomings such as difficulty in maintaining the worms' viability in the culture, poor RNAi trigger delivery, and compatibility issues of the culture medium with different life stages of the worm [25]. To overcome these drawbacks of in vitro protocols, in our study an in vivo approach was developed, where $S$. digitata larval stages were cultured in its intermediate host, Culex quinquefasciatus, and the RNAi trigger (siRNA) was directly injected into the mosquito [19], and the possibility of using the mosquito as a better siRNA delivery platform examined. Furthermore, we focused on developing an effective RNAi protocol to specifically shut down the gene of interest to study its function and explore the gene's potential as a likely drug target.

\section{Methods}

\section{Mosquito colony establishment}

Culex quinquefasciatus were collected from a cattle trap and maintained at $27{ }^{\circ} \mathrm{C}$ with $80 \%$ relative humidity, with cycles of $16 \mathrm{~h}$ of light and $8 \mathrm{~h}$ of darkness, in $12 \times$ $12 \times 12$-inch cages [26]. The eggs obtained from these mosquitoes were hatched in cups containing pre-boiled water, and the larvae which emerged from the eggs were fed with a larval diet (brewer yeast, bovine liver powder, tuna meal) until pupae appeared. The pupae were then separated from the larvae and placed in containers with fresh water in separate cages for $36 \mathrm{~h}$ until adult mosquitoes emerged. These were then fed with $10 \%$ sucrose solution [27]. They were used as an in vivo culturing medium for $S$. digitata microfilariae.

\section{Mosquito infection with S. digitata}

Adult female worms obtained from a cattle slaughterhouse in Welisara, Ragama, Sri Lanka were cut into small pieces after washing three times with mosquito physiological saline (MPS) [28] to release microfilariae (MF) into a tube containing $300 \mu \mathrm{l}$ MPS. This was vortexed and centrifuged at $1500 \times \mathrm{rpm}$ for $30 \mathrm{~s}$, and the supernatant recovered was then re-centrifuged at $1500 \times$ rpm for 3 min to obtain a pellet containing MF, which was then re-suspended in MPS to achieve a microfilariae (MF) concentration of $40 \mathrm{MF} / \mu \mathrm{l}$.

The thorax of anaesthetized adult mosquitoes (aged 3 days) on ice [29] was injected with a volume of $0.5 \mu \mathrm{l}$ of microfilariae suspension using a pre-pulled borosilicate glass capillary attached to a manual injector [29-31]. The injected mosquitoes were kept in paper cups covered with a net while providing them with cotton pads soaked with the sucrose solution.

\section{siRNA synthesis, Cy3 labelling and RNAi treatment}

The entire coding sequence of SDNP was divided into four overlapping regions, and each of them was amplified by separate PCR reactions using four separate 
primer sets containing T7 promoter sequence at $5^{\prime}$ ends (Table 1) to enrich the total yield of the template and PCR products of overlapping regions were used to synthesize siRNA. The PCR conditions used for the amplification were initial denaturation at $94{ }^{\circ} \mathrm{C}$ for 2 min, followed by 40 cycles of denaturation at $94{ }^{\circ} \mathrm{C}$ for $30 \mathrm{~s}$, annealing at $55^{\circ} \mathrm{C}$ for the primer sets SDNP-1-F/R, SDNP-2-F/R and SDNP-3-F/R and $45^{\circ} \mathrm{C}$ for the primer set SDNP-4-F/R, and extension at $72{ }^{\circ} \mathrm{C}$ for $30 \mathrm{~s}$ with a final extension of $10 \mathrm{~min}$ at $72{ }^{\circ} \mathrm{C}$ (Table 1 ).

The resulting PCR fragments were transcribed separately in vitro by T7 RNA polymerase (New England Biolabs, Hitchin, UK) using slightly modified manufacturer's protocols, to yield a high amount of dsRNA. Briefly, the in vitro transcription reaction was carried out overnight at $37{ }^{\circ} \mathrm{C}$ in a $120 \mu \mathrm{l}$ reaction mix containing $81.5 \mu \mathrm{l}$ of nuclease-free $\mathrm{H}_{2} \mathrm{O}, 12.0 \mu \mathrm{l}$ of $10 \times$ T7 buffer, $3.0 \mu \mathrm{l}$ of DTT $(100 \mathrm{mM}), 10 \mu \mathrm{l}(2 \mu \mathrm{g})$ of PCR product, $12 \mu \mathrm{l}$ of rNTP mix $(80 \mathrm{mM}), 1.5 \mu \mathrm{l}$ of T7 RNA polymerase $(18 \mathrm{U} / \mu \mathrm{l})$. The RNA products of in vitro transcribed DNA were first treated with DNase 1 (New England BioLabs) to remove DNA templates and then incubated at $75{ }^{\circ} \mathrm{C}$ for $5 \mathrm{~min}$ before cooling them down to room temperature for the complementary strands of synthesized RNA to anneal to make dsRNA. The dsRNA (assessed by gel electrophoresis) was digested with RNase III (New England Biolabs) according to the manufacturer's protocol to produce 2123 mer siRNA. They were analyzed by polyacrylamide gel electrophoresis before labelling with Cy3 fluorescent dye using a Silencer ${ }^{\circ}$ siRNA Labeling Kit - $\mathrm{Cy}^{\mathrm{max}} 3$ dye (Ambion, Life Technologies, California, USA) according to the manufacturer's instructions [32].

The ds-RNA synthesized from the gene encoding for the green fluorescent protein (GFP) of Aequorea victoria was also used as the non-specific siRNA control as this gene neither has any homologous sequences in S. digitata nor mosquito. pCRII TOPO vector (pCRII TOPO-GFP) containing GFP was used in the synthesis of GFP dsRNA. The vector was digested with Kpn I [7.5 $\mu$ l nuclease free
$\mathrm{H}_{2} \mathrm{O}, 2.0 \mu \mathrm{l} 10 \times$ buffer, $0.5 \mu \mathrm{l} \mathrm{Kpn} \mathrm{I} \mathrm{(15} \mathrm{U/ \mu l),} 10 \mu \mathrm{l}$ DNA $(200 \mathrm{ng} / \mu \mathrm{l})$; incubated $1 \mathrm{~h}$ at $\left.37^{\circ} \mathrm{C}\right]$ and Xho I [10 $\mu \mathrm{l} \mathrm{nu}$ clease free $\mathrm{H}_{2} \mathrm{O}, 3.0 \mu \mathrm{l} 10 \times$ buffer, $0.3 \mu \mathrm{lBSA}, 0.5 \mu \mathrm{l}$ Xho I $(20 \mathrm{U} / \mu \mathrm{l}), 16.2 \mu \mathrm{l}$ DNA $(200 \mathrm{ng} / \mu \mathrm{l})$; incubated $3 \mathrm{~h}$ at $37^{\circ}$ C] separately. The Kpn I digested vector was in vitro transcribed by T7 RNA polymerase using the same protocol indicated above. The Xho I digested vector was in-vitro transcribed by Sp6 RNA polymerase using the following reaction; $60 \mu \mathrm{l}$ of nuclease-free $\mathrm{H}_{2} \mathrm{O}, 8.0 \mu \mathrm{l}$ of $10 \mathrm{x}$ Sp6 buffer, $2 \mu \mathrm{g}(10 \mu \mathrm{l})$ of pCR II-TOPO vector, $8 \mu \mathrm{l}$ of rNTP mix $(80 \mathrm{mM}), 2 \mu \mathrm{l}$ of Sp6 RNA polymerase $(20 \mathrm{U} / \mu \mathrm{l})$, incubated at $40^{\circ} \mathrm{C}$ for $4 \mathrm{~h}$. The separately in vitro transcribed RNA was mixed and incubated at $75{ }^{\circ} \mathrm{C}$ for $5 \mathrm{~min}$ and cooled down to room temperature for the complementary strands of synthesised RNA to anneal to make dsRNA.

The $S$. digitata infected mosquitoes were initially injected with $150 \mathrm{ng}$ of Cy3 labelled SDNP siRNA mix into their thorax at $12 \mathrm{~h}$ post infection (hpi) to monitor the uptake of siRNA by larvae. To target the L2 to L3 larvae transformation, non-labelled siRNA mix was injected at eight days post infection (dpi). The GFP siRNA (150 ng) was injected as a negative control.

\section{Mosquito dissection}

The mosquitoes infected with $S$. digitata and injected with SDNP siRNA, non-injected, GFP siRNA-injected and buffer-injected were dissected at different time intervals (24 hpi, 8-14 dpi) under a dissection microscope. In this process, the bodies of cold anaesthetized mosquitoes were separated into the thorax, midgut, and head, and each of these body parts was further dissected to release the larvae on to glass slides. The MF obtained from the smeared content on the slides at 24 hpi were visualized under the fluorescent microscope using Cy3 filter set (excitation: $550 \mathrm{~nm}$, emission: $570 \mathrm{~nm}$ ) to detect the uptake of siRNA. The transformation of L2 larvae to L3 was recorded from the mosquitoes dissected at 8 to 14 $\mathrm{dpi}$, and the following data were recorded only from the mosquitoes dissected at 14 dpi: (i) larvae length and

Table 1 Primer sets used for the synthesis of dsRNA. The positions of the primers with respect to SDNP sequence (GenBank: GU222920), sequence corresponding to T7 promoter region of primers (underlined), the size of the PCR products, and the optimized annealing temperatures of primer sets are given

\begin{tabular}{|c|c|c|c|c|}
\hline Primer & Position & Sequence $\left(5^{\prime}-3^{\prime}\right)$ & Product size (bp) & Annealing $T\left({ }^{\circ} \mathrm{C}\right)$ \\
\hline$\overline{S D N P-1-F}$ & 1 & $\begin{array}{l}\text { TAATACGACTCACTATAGGGCGACGAGGGTTCCATTGAGTGA } \\
\end{array}$ & 154 & 55 \\
\hline SDNP-1-R & 134 & TAATACGACTCACTATAGGGGCTTCCTGACAAGCCAAACAT & & \\
\hline SDNP-2-F & 155 & $\underline{\text { TAATACGACTCACTATAGGGCGACGAGGGTTCCATTGAGTGA }}$ & 150 & 55 \\
\hline SDNP-2-R & 282 & TAATACGACTCACTATAGGGTTGGAATATAAACATGCGGTATA & & \\
\hline SDNP-3-F & 305 & TAATACGACTCACTATAGGGCTACCGGTATCAGAACTCAGAA & 157 & 55 \\
\hline SDNP-3-R & 462 & TAATACGACTCACTATAGGGTAGCGCTGGACCGAATTCTTाT & & \\
\hline SDNP-4-F & 463 & TAATACGACTCACTATAGGGGGAAATGGAACTTGTGAAATAA & 155 & 45 \\
\hline SDNP-4-R & 618 & TAATACGACTCACTATAGGGTCAGTAATTAATCAAATT & & \\
\hline
\end{tabular}


Table 2 Primers used for qPCR. The primer sequences of the target (SDNP) and internal control (Actin) are given

\begin{tabular}{|c|c|c|c|c|}
\hline Primer & Sequence $\left(5^{\prime}-3^{\prime}\right)$ & Position & Product size (bp) & Annealing $T\left({ }^{\circ} \mathrm{C}\right)$ \\
\hline \multicolumn{5}{|l|}{ SDNP amplification } \\
\hline SDNP-RTPCR-FP & AAGACTTGCAACTCCATCTCGAT & 219 & 170 & 60 \\
\hline SDNP-RTPCR-RP & ATTTCTCGCTGACCACCACAACT & 366 & & \\
\hline \multicolumn{5}{|c|}{ S. digitata actin amplification (reference gene) } \\
\hline SDACT-RTPCR-FP & CGCTCGAGAAATCTTACGAATTGC & 707 & 147 & 55 \\
\hline SDACT-RTPCR-RP & TCCTITCTGATATCGATATCACATT & 854 & & \\
\hline
\end{tabular}

\section{Abbreviation: $T$ temperature}

width (Image $\mathrm{J}$ software was utilized in obtaining the measurements); (ii) larvae location in the mosquito (larvae in the head, thorax and abdomen of the mosquito; (iii) larvae motility (motility of the RNAi treated and control worms were compared according to a previously used pre-fashioned 5-point scheme based on Song et al. [19]). The scoring scheme was as follows: 1 , immobile, dead; 2, compromised motility; 3, partial movement of the body; 4, slightly reduced motion in the body; and 5, all parts of the body are vigorously twisting. Values in

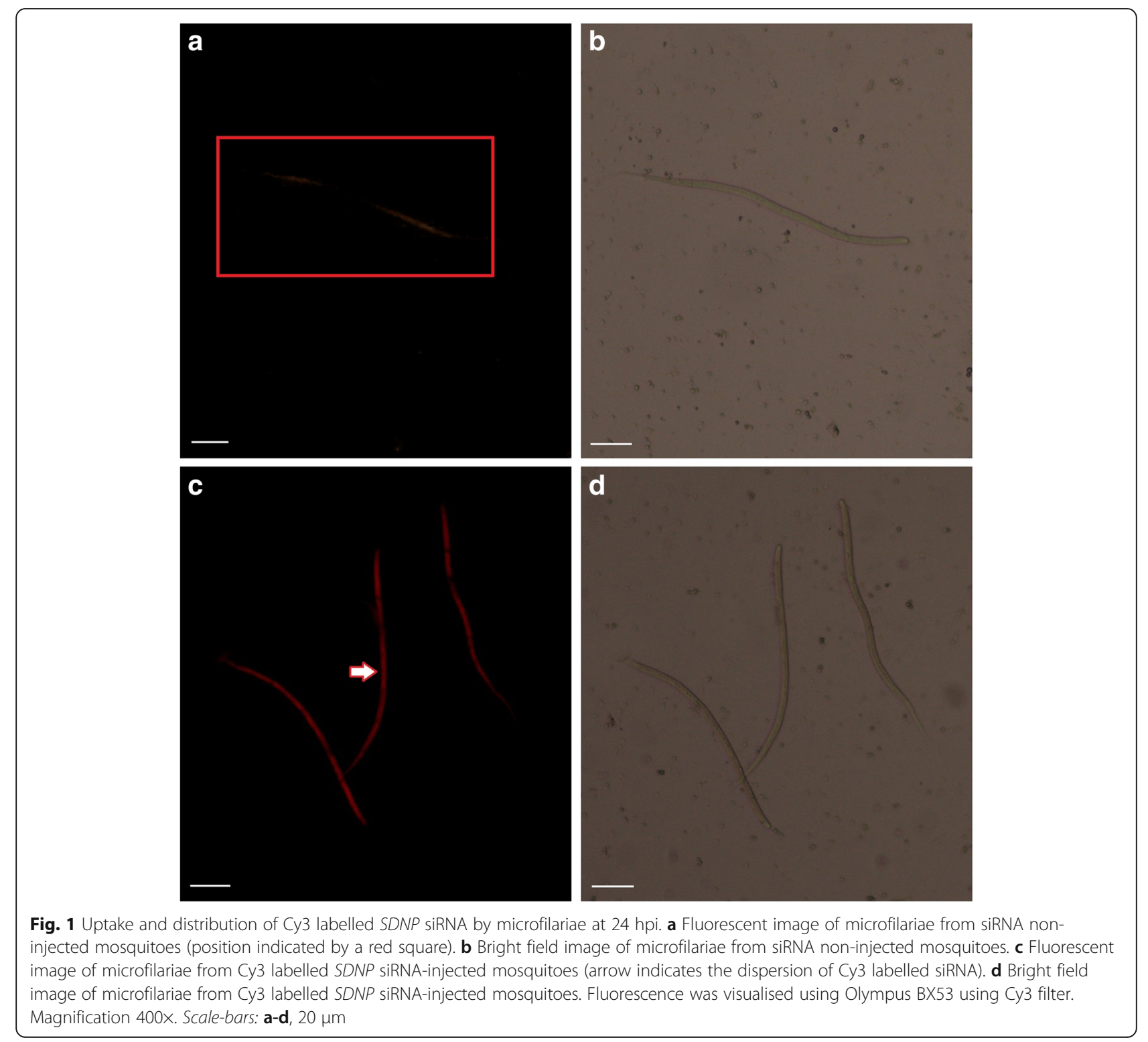



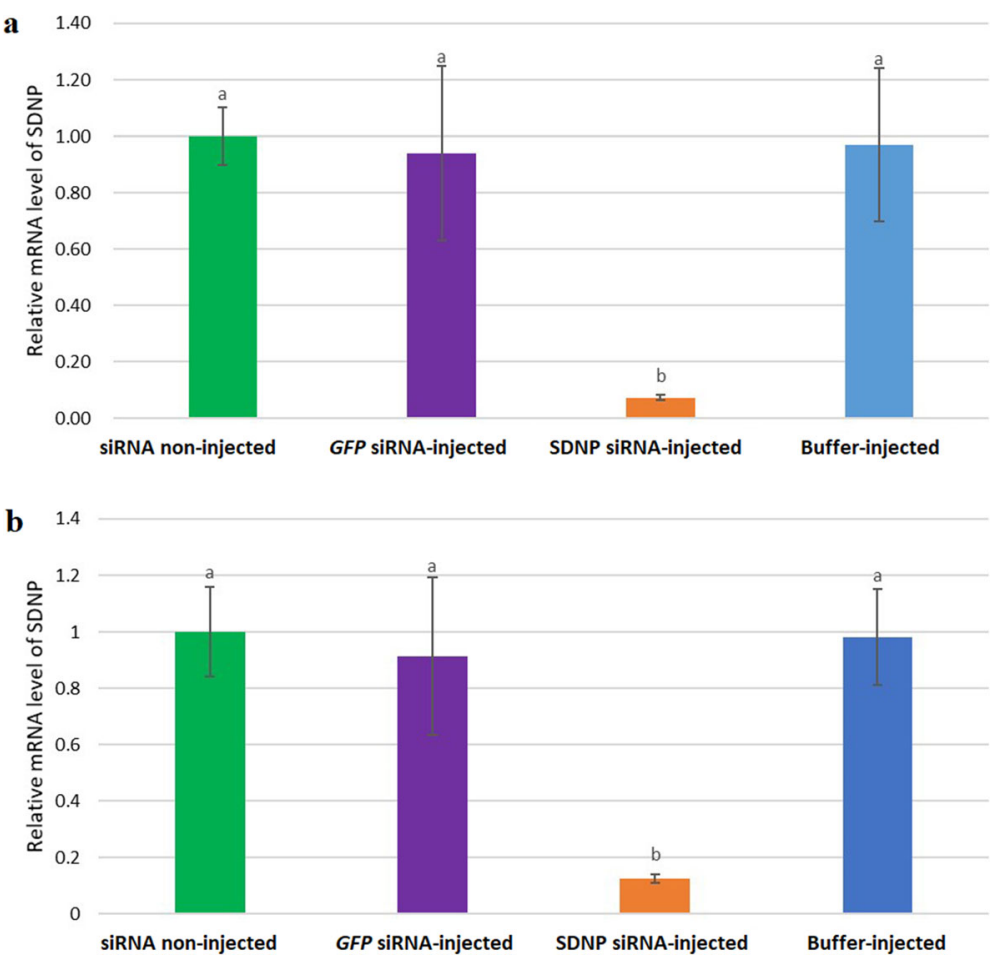

Fig. 2 Relative mRNA expression changes owing to the RNAi. a Relative mRNA levels of SDNP at two dpi. b Relative mRNA levels of SDNP at 14 dpi. Changes of SDNP specific transcript levels were analysed by $\mathrm{QPCR}$, and the $2^{-\triangle \triangle C T}$ method was used in evaluating the results. For the reference gene, S. digitata actin gene was used. GFP was used as the non-specific control. SDNP transcript level was presented as relative to that of non-treated worms. Each experiment was performed in triplicate. Bars display mean \pm SD. Statistical analysis was performed using Student's ttest $(P<0.05)$. Significant differences are represented by different lowercase letters

between 5 and 1 in the scale were assigned according to the decreasing motility blindly by an independent evaluator; (iv) at $14 \mathrm{dpi}$, mosquito survival rate was also measured by counting the live mosquitoes.

\section{Relative quantification of SDNP mRNA level}

Total RNA was extracted at two dpi and 14 dpi from non-injected, GFP siRNA injected, SDNP siRNA-injected and buffer-injected mosquitoes infected with S. digitata using a GenoSpin $\mathrm{R}^{\mathrm{TM}}$ Total RNA Extraction Kit (CEYGEN Biotech, Colombo, Sri Lanka) according to the manufacturer's instructions. Five mosquitoes were used from each batch. DNA contamination in extracted RNA was removed by treating with DNase I and heat inactivating the DNase I at $75^{\circ} \mathrm{C}$ for $5 \mathrm{~min}$. cDNA synthesis was carried out using RNA $(2 \mu \mathrm{g})$ purified using an RNeasy Plus Mini Kit (Qiagen, California, USA) in a $20 \mu \mathrm{l}$ reaction mix containing $5.0 \mu \mathrm{l}$ of M-MLV transcription buffer, $1.0 \mu$ l of oligo dT primers $(5 \mathrm{nM}), 1.25 \mu \mathrm{l}$ of dNTP mix $(10 \mathrm{nM}), 1.0 \mu \mathrm{l}$ RNase inhibitor (20 U), 1.0 $\mu \mathrm{l} \mathrm{M-MLV} \mathrm{reverse} \mathrm{transcriptase}$ (200 U, Promega, California, USA), RNA ( $2 \mu \mathrm{g})$, and RNase free water according to the vendor's protocol.

Relative quantification of SDNP mRNA levels was carried out by quantitative real-time PCR [CFX96 RealTime PCR System (Bio-Rad)] using SYBR green as the DNA binding dye. qPCR conditions were initial denaturation at $95{ }^{\circ} \mathrm{C}$ for $2 \mathrm{~min}$, followed by 40 cycles of denaturation at $95{ }^{\circ} \mathrm{C}$ for $30 \mathrm{~s}$ annealing at $60{ }^{\circ} \mathrm{C}$ for SDNP primers and $55{ }^{\circ} \mathrm{C}$ for actin primers for $45 \mathrm{~s}$ (Table 2), and with an extension at $72{ }^{\circ} \mathrm{C}$ for $30 \mathrm{~s}$. In qPCR, the actin gene (GenBank: AF079359.1) was used as the internal amplification control in a separate tube for each qPCR reaction. In calculating the relative mRNA expression level, the $2^{-\triangle \Delta C T}$ method was used as mentioned in qPCR Application Guide (BioRad). The data obtained were presented as the mean \pm standard deviation (SD). A $P$ value $<0.05$ was considered statistically significant.

\section{Results and discussion}

siRNA uptake by $S$. digitata microfilariae in the mosquito host

A previous study demonstrated the possibility of in vivo suppression of the cathepsin L-like cysteine protease gene of B. malayi using RNAi in Aedes aegypti [19]. Therefore, in this study, the possibility of using $C$. quinquefasciatus as an in vivo siRNA delivery platform for developing $S$. digitata larvae was tested, where the transformation of MF to L3 larvae of the latter organism has been observed. Since the previously stated study revealed 

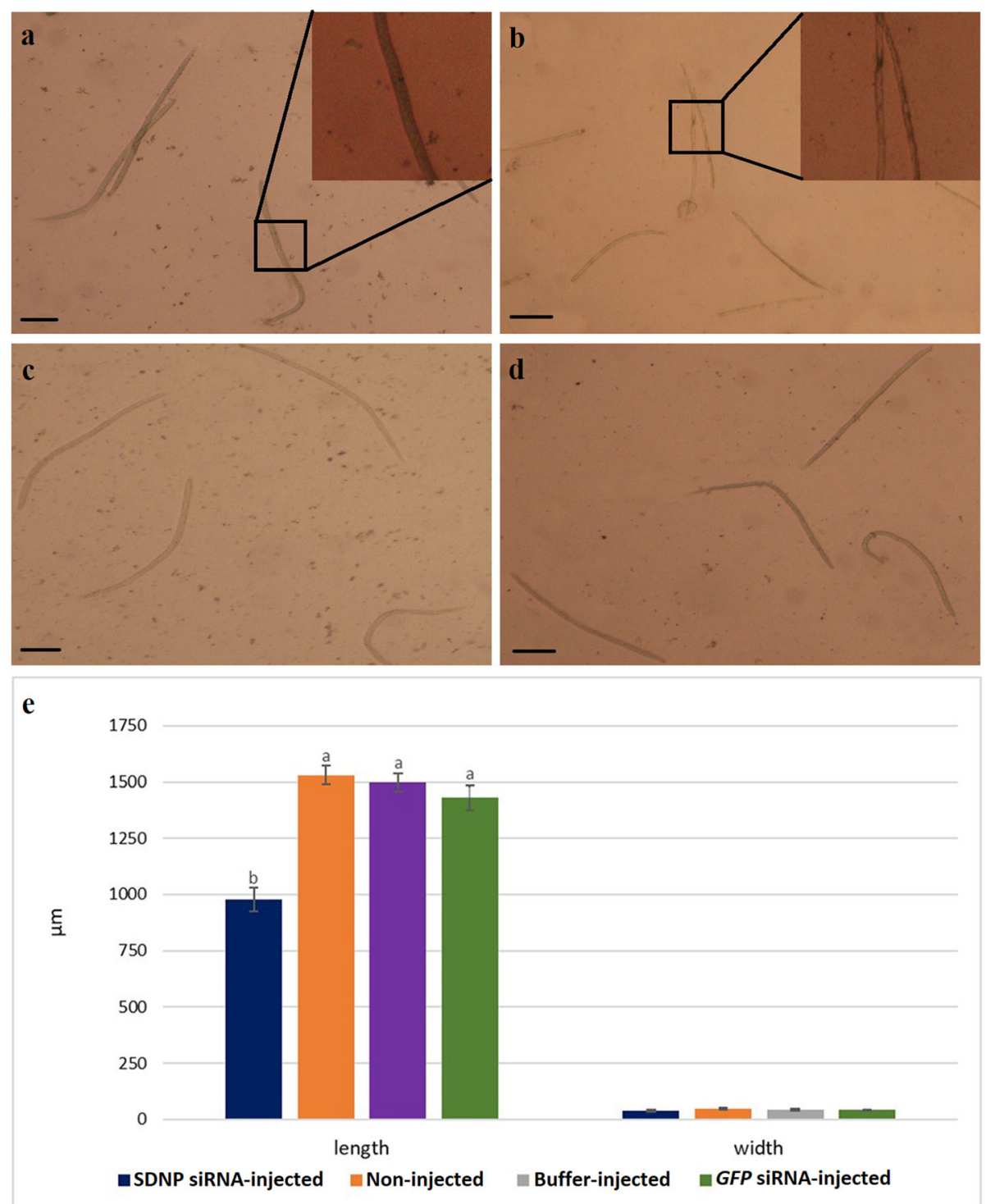

Fig. 3 Larvae development in C. quinquefasciatus mosquitoes at 14 dpi. a Larvae in siRNA non-injected mosquito. b Larvae in SDNP siRNAinjected mosquito. c Larvae in GFP siRNA-injected mosquito. $\mathbf{d}$ Larvae in buffer-injected mosquito (magnification 400x). e Mean length and width of larvae form SDNP siRNA-injected, non-injected, buffer-injected and GFP siRNA-injected mosquitoes. To target L2 to L3, siRNA injections were made at $8 \mathrm{dpi}$. Triplicates were performed for each experiment. Bars represent mean \pm SD. Significant differences at $P<0.05$ are represented by different lowercase letters. Scale-bars: a-d, $400 \mu \mathrm{m}$

the dissemination of siRNA to the MF of B. malayi through the mosquito, in the current study the uptake of the Cy3 labeled RNAi trigger by MF was investigated by, comparing the internal fluorescence of the MF with the mosquito physiological saline (MPS) injected control under a fluorescent microscope (Fig. 1a). A dispersion of the fluorescent signal was observed throughout the body of the tested MF at $24 \mathrm{hpi}$ (Fig. 1c) indicating that uptake of $\mathrm{Cy} 3$ labelled siRNA by $\mathrm{MF}$ is occurring in the mosquitoes. Although the mechanism of siRNA uptake is unclear at this point, it is possible that this would occur via feeding or through the cuticle of MF. Therefore, here, it was possible to recreate the in vitro siRNA soaking conditions for S. digitata larvae in vivo, and the possibility of utilizing the mosquito as a siRNA delivering platform for parasitic nematodes hosted by the mosquito was affirmed.

\section{The effect of siRNA on SDNP mRNA level of MF}

Several recent studies involving RNAi on nematodes showed that genes could be specifically suppressed by soaking the nematode in in vitro culture media consisting of RNAi triggers [25, 32-35]. In this study, instead 


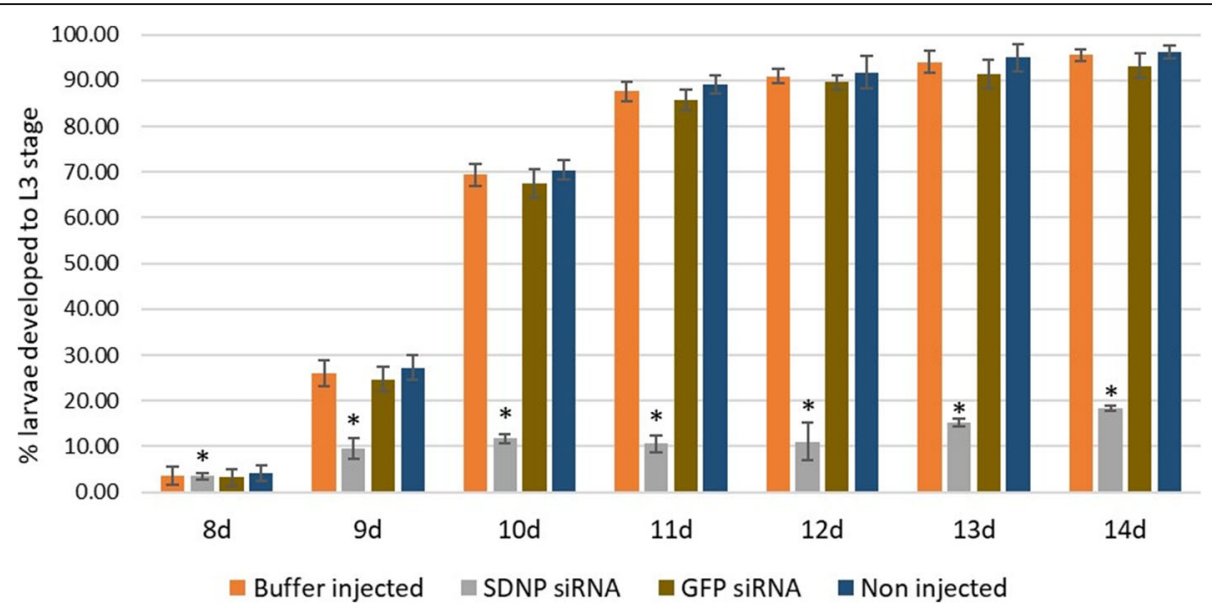

Fig. 4 Percentage of larval development to L3 stage out of total larvae inside the mosquito. S. digitata larvae from non-injected, SDNP siRNAinjected, GFP siRNA-injected and buffer-injected C. quinquefasciatus are represented by four different patterns in the graph. To target L2 to L3, siRNA injections were made at $8 \mathrm{dpi}$. Triplicates were performed for each experiment. Bars represent mean $\pm \mathrm{SD}$. Significant differences at $P<$ 0.05 are indicated by *

of using the in vitro culture conditions, we recreated the soaking conditions in vivo by injecting the mosquitoes harbouring nematode larvae with siRNA. The RNAi effect on SDNP transcript level was assessed two dpi, and 14 dpi of siRNA using qPCR and the knockdown of the gene was determined by comparing the transcript levels of the worms derived from non-injected, buffer-injected and GFP siRNA-injected mosquitoes. A significant suppression of the target gene expression was observed in this study, and the transcript abundance of the SDNP was reduced by $93 \%$ (unpaired two-tailed t-test, $t_{44}=15.8716, P=0.05$ ) and $87.4 \%$ (unpaired two-tailed t-test, $\left.t_{44}=15.2220, P=0.05\right)$ in the worms from $S D N P$ siRNA-injected mosquitoes, compared to the non-injected at $2 \mathrm{dpi}$ and $14 \mathrm{dpi}$, respectively, compared to worms from non-injected mosquitoes (Fig. 2a, b). Worms from the buffer-injected, and GFP siRNA-injected mosquitoes displayed no significant changes in the SDNP transcript levels (Fig. 2a, b). The qPCR results obtained proved the worms' susceptibility to specific RNAi while showing the effectiveness of the new siRNA delivery platform developed in this study and the capability of siRNA to knockdown the targeted gene specifically in this parasitic nematode.

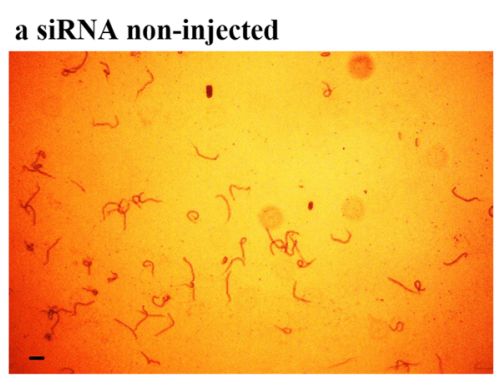

\section{b SDNP siRNA-injected}

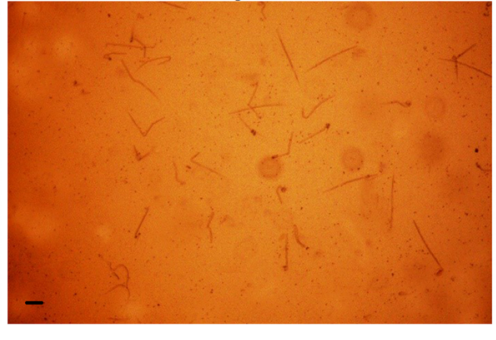

c GFP siRNA-injected

d Buffer-injected
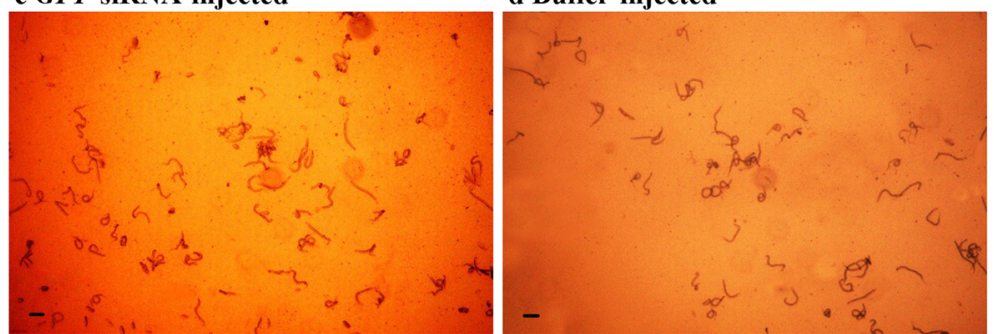

Fig. 5 Motility comparison of larvae at 14 dpi in, a non-injected, b SDNP siRNA-injected, c GFP siRNA-injected, $\mathbf{d}$ buffer-injected mosquitoes (Magnification 40x) Scale-bars: a-d, $400 \mu \mathrm{m}$ 
a siRNA non-injected

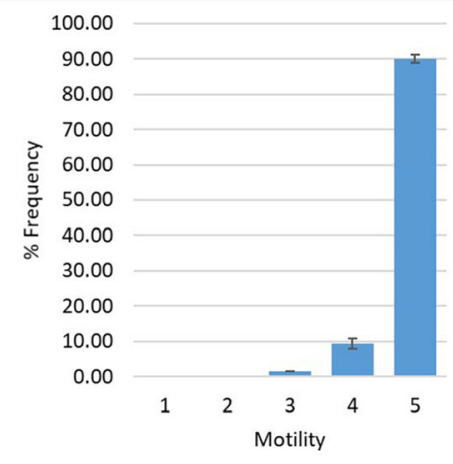

\section{c GFP siRNA-injected}

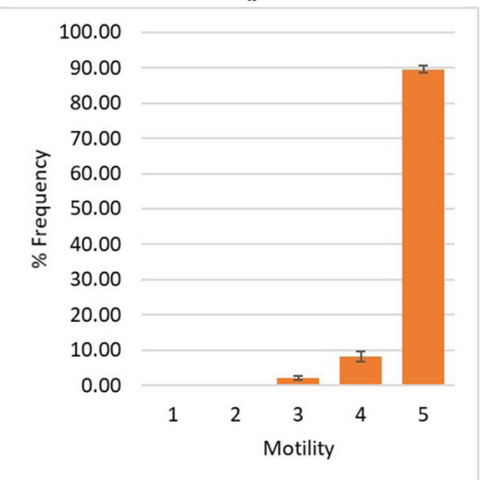

b SDNP siRNA-injected

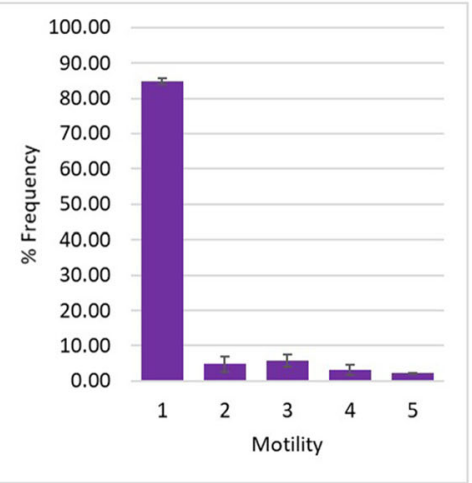

\section{d Buffer-injected}

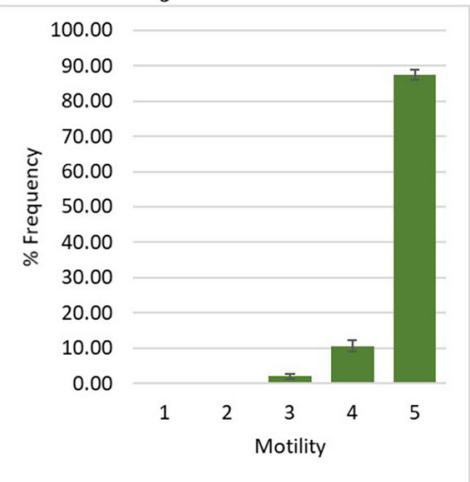

Fig. 6 Frequency distribution for motility of S. digitata larvae at 14 dpi in, a siRNA non-injected, b SDNP siRNA-injected, c GFP siRNA-injected and d buffer-injected mosquitoes. The scoring scheme was: 1, immobile or dead; 2, compromised motility; 3, partial movement of the body; 4, slightly reduced motion in the body; 5 , all parts of the body are vigorously twisting. Values in between 5 and 1 in the scale were assigned according to the decreasing motility blindly by an independent evaluator. Five replicates were performed for each experiment. Bars represent mean \pm SD

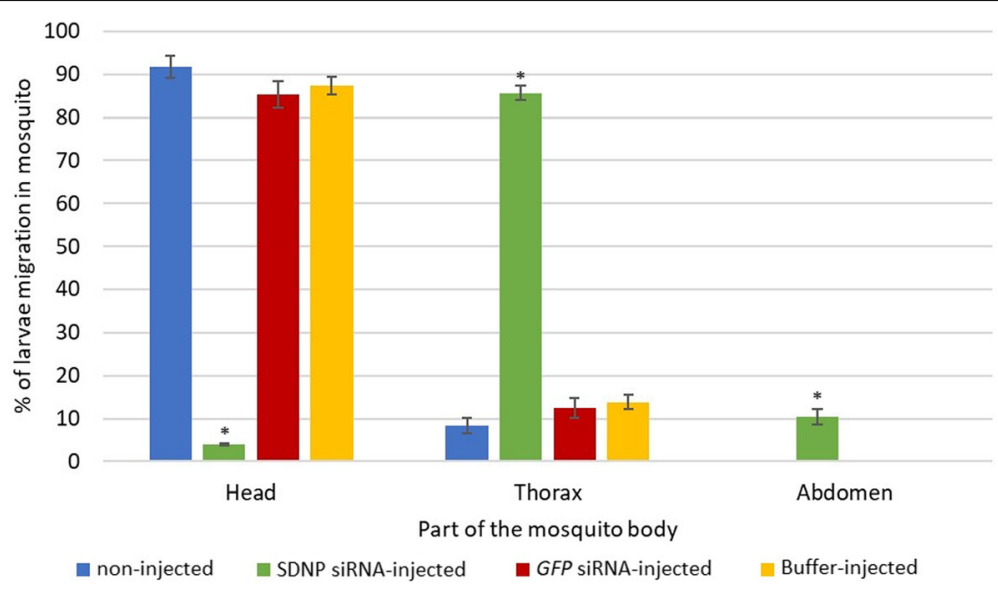

Fig. 7 Frequency distribution of S. digitata larvae in C. quinquefasciatus head, thorax, or abdomen. Migration of the larvae was monitored at 14 dpi in non-injected, SDNP siRNA-injected, GFP siRNA-injected and buffer-injected mosquitoes. Mosquitoes were dissected into head, thorax and abdomen and larvae in each part was counted. Five replicates were performed for each experiment. Bars represent mean \pm SD. Statistical analysis was performed using Student's t-test $(P<0.05)$. Significant differences at $P<0.05$ are indicated by * 


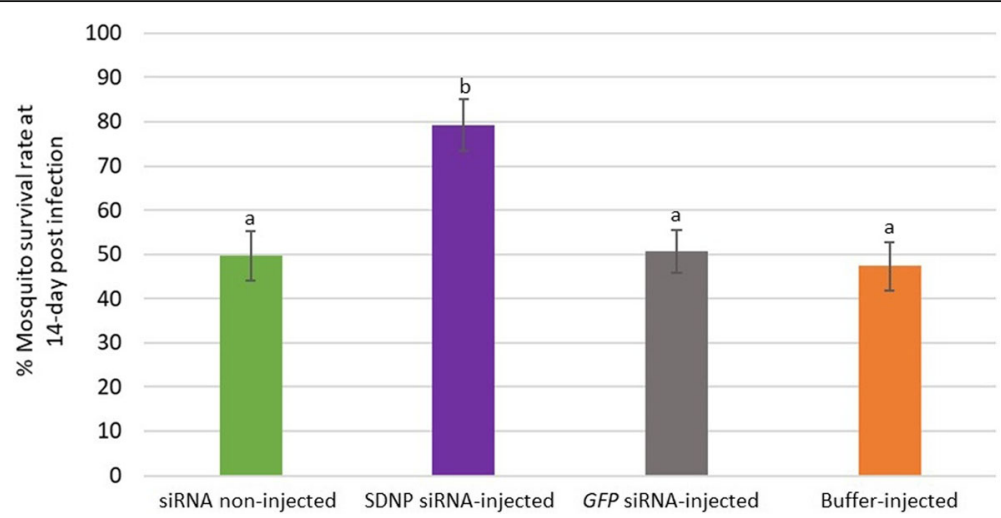

Fig. 8 Mosquito survival rate. Survivability of the non-injected, SDNP siRNA-injected, GFP siRNA-injected and buffer-injected mosquitoes at 14 dpi was measured. Five replicates were performed for each experiment. Bars represent mean $\pm \mathrm{SD}$. Significant differences at $P<0.05$ are indicated with different lowercase letters

\section{Changes in developmental phenotypes and motility of larvae because of RNAi}

To study the phenotypic changes, if any, associated with the transformation of $\mathrm{L} 2$ to $\mathrm{L} 3$ in the SDNP siRNA-injected group in comparison to the control mosquito groups, the infected mosquitoes were dissected to recover the larval stages at eight dpi to 14 dpi following the RNAi trigger. A significant reduction in body length and width together with deformities in the body structure was observed in the larvae from SDNP siRNA-injected mosquitoes at $14 \mathrm{dpi}$ (Fig. 3b, e) compared to the non- injected, buffer-injected and GFP siRNA-injected counterparts (Fig. 3a, c-e). In addition, aberrations, with larval lethality was also observed in SDNP siRNA treated larvae compared to its control groups, and with $\sim 96 \%$ of L2 to L3 transformation in non-treated, $\sim 95 \%$ in buffer-treated and $\sim 93 \%$ in GFP siRNA treated compared to $\sim 18 \%$ of such transformation in treated at $14 \mathrm{dpi}$ (Fig. 4). The L2 and L3 larvae were differentiated based on their length of the body. No significant developmental changes were observed in the worms from GFP siRNA-injected mosquitoes suggesting the phenotypic changes are specific to SDNP siRNA and not due to the injection of non-specific siRNA. Therefore, it is reasonable to assume these phenotypic changes are most likely due to the downregulation of SDNP transcripts, which in turn affects the larval development. The previous finding reported by us, i.e., the ubiquitous expression of SDNP in all life stages of S. digitata and also its predominant expression in longitudinal muscle, reproductive systems, and developing embryos [24, 36], together with the findings of the present study, i.e. association of SDNP downregulation with irregularities in the larvae development, transformation arrest, changes in phenotypes, etc. in larvae can be used to further strengthen our previous suggestion regarding the involvement of SDNP in growth and development of nematodes.

In the motility changes monitored at $14 \mathrm{dpi}$ of the control worms and worms from SDNP siRNA-injected mosquitoes, the former displayed a vigorously convoluting body movement (Fig. 5a, c, d) of 4 and 5 in the motility scale (Fig. 6a, c, d) while the SDNP siRNA-treated showed a significantly depressed body movement, feeble body twitching, and total body paralysis (Figs. 5b, 6b). Also, the worms from SDNP siRNA-injected worms and monitored at 14 dpi displayed irregular migration patterns in the mosquitoes compared to the controls (Fig. 7). Worms from the control mosquitoes were predominantly found in the head (non-injected: 91\%; GFP siRNA-injected: $\sim 85 \%$; buffer-injected: $\sim 87 \%$ ) of the mosquito, while $S D N P$-suppressed worms were mainly found either in the thorax $(\sim 85 \%)$ or abdomen $(\sim 10 \%)$ indicating that motility irregularities of larvae are associated with the migration within the mosquito. It has been previously revealed that $S D N P$ had homology with the inter-domain linkers of the muscle-specific twitchin kinase of $C$. elegans and was expressed in the longitudinal muscles of the adult worm [24]. Hence, the downregulation of the SDNP could affect the muscle movement of the developing worm, which in turn reduces the migration of the parasite in the mosquito. The increased mosquito survival rate was seen in the SDNP siRNA-injected mosquito group compared to the control groups (Fig. 8). This observation suggests that the compromised motility and development of the larvae induced by the SDNP siRNA treatment could reduce the parasitic burden on the mosquito, thereby increasing its survival.

\section{Conclusions}

In this study, we demonstrated the successful usage of the RNAi approach to turn off the expression of SDNP in the larval stages of $S$. digitata in their natural milieu, 
C. quinquefasciatus, and the possibility of using this host as an in vivo culture platform in the absence of proper culture conditions to study the biological functions of the genes that the $S$. digitata genome encodes. Further, we showed that the specific silencing of SDNP is associated with developmental deformities and motility changes in the developing larvae of S. digitata, in addition to the reduction of migration of the larvae to the head of the mosquito. Therefore, by taking the outcomes of present and our previous studies into consideration, it can be concluded that SDNP plays a vital role in the movement and development of larvae. Hence, it can be suggested that the SDNP may be utilised as a candidate anthelminthic drug target.

\section{Abbreviations}

RNAi: RNA interference; SDNP: Setaria digitata novel protein; qPCR: Quantitative real-time polymerase chain reaction; siRNA: Small interfering RNA; dsRNA: Double stranded RNA; MPS: Mosquito physiological saline; MF: Microfilariae; hpi: Hour post infection; dpi: Days post-infection; GFP: Green fluorescent protein.

\section{Acknowledgement}

The corresponding author wishes to acknowledge Professor Mark Taylor and his team for providing him training in RNA interference at his laboratories in Liverpool School of Tropical Medicine, Liverpool under the Commonwealth Fellowship Programme in 2012.

\section{Funding}

The research was funded by a grant (AP/3/2011/CG/03) awarded to Professor R.S. Dassanayake by the University of Colombo, Sri Lanka, to promote collaborative research. The funders had no role in study design, data collection and analysis, decision to publish, or preparation of the manuscript.

\section{Availability of data and materials}

The data supporting the conclusions of this study are included within the article. Clones and raw data are available from the authors upon request.

\section{Authors' contributions}

MBCLS carried out research work, collected samples, analysed data, and wrote the manuscript. YINSG supervised research work, analysed research data and wrote the manuscript. NVC supervised research work data and corrected the manuscript. ANBE analysed research data and corrected the manuscript. RSD designed and supervised the study and corrected the manuscript. All authors read and approved the final manuscript.

\section{Ethics approval and consent to participate}

Not applicable.

\section{Consent for publication}

Not applicable.

\section{Competing interests}

The authors declare that they have no competing interests.

\section{Publisher's Note}

Springer Nature remains neutral with regard to jurisdictional claims in published maps and institutional affiliations.

\section{Author details}

'Department of Chemistry, Faculty of Science, University of Colombo, Colombo 03 00300, Sri Lanka. ${ }^{2}$ Molecular Medicine Unit, Faculty of Medicine, University of Kelaniya, Ragama 11010, Sri Lanka. ${ }^{3}$ Department of Bioclinical Sciences, Faculty of Dentistry, Health Sciences Center, University of Kuwait, Kuwait City, Kuwait.
Received: 10 May 2018 Accepted: 4 September 2018

Published online: 10 October 2018

\section{References}

1. Bhadury P, Austen MC, Bilton DT, Lambshead PJD, Rogers AD, Smerdon GR. Development and evaluation of a DNA-barcoding approach for the rapid identification of nematodes. Mar Ecol Prog Ser. 2006;320:1-9.

2. Stepek G, Buttle DJ, Duce IR, Behnke JM. Human gastrointestinal nematode infections: are new control methods required? Int J Exp Pathol. 2006;87:325-41.

3. Global Programme to Eliminate Lymphatic Filariasis. Progress report on mass drug administration 2010. Switzerland: World Health Organization; 2011.

4. Singh S, Singh B, Singh AP. Nematodes: a threat to sustainability of agriculture. Procedia Environ Sci. 2015;29:215-6.

5. Roeber F, Jex AR, Gasser RB. Impact of gastrointestinal parasitic nematodes of sheep, and the role of advanced molecular tools for exploring epidemiology and drug resistance - an Australian perspective. Parasit Vectors. 2013;6:153.

6. Gilleard JS. Understanding anthelmintic resistance: the need for genomics and genetics. Int J Parasitol. 2006;36:1227-39.

7. Kaplan RM. Drug resistance in nematodes of veterinary importance: a status report. Trends Parasitol. 2004;20:477-81

8. Richard-Lenoble D, Chandenier J, Gaxotte P. Ivermectin and filariasis. Fundam Clin Pharmacol. 2003;17:199-203.

9. Berriman M, Lustigman S, McCarter JP. Genomics and emerging drug discovery technologies. Expert Opin Drug Discov. 2007;2:S83-9.

10. Foster JM, Zhang Y, Kumar S, Carlow CKS. Mining nematode genome data for novel drug targets. Trends Parasitol. 2005;21:101-4.

11. Kuwabara PE, Coulson A. RNAi - prospects for a general technique for determining gene function. Parasitol Today. 2000;16:347-9.

12. Allshire R. Retraction. Hairpin RNAs and retrotransposon LTRs effect RNAi and chromatin-based gene silencing. Science. 2005;310:49.

13. Hall IM, Shankaranarayana GD, Noma K-I, Ayoub N, Cohen A, Grewal SIS Establishment and maintenance of a heterochromatin domain. Science. 2002;297:2232-7.

14. Behm CA, Bendig MM, McCarter JP, Sluder AE. RNAi-based discovery and validation of new drug targets in filarial nematodes. Trends Parasitol. 2005; 21:97-100.

15. Fire A, Albertson D, Harrison SW, Moerman DG. Production of antisense RNA leads to effective and specific inhibition of gene expression in $C$. elegans muscle. Development. 1991;113:503-14.

16. Grant WN, Behm CA. Target identification and validation for anthelmintic discovery. Expert Opin Drug Discov. 2007;2:S91-8.

17. Pfarr $\mathrm{K}$, Heider $U$, Hoerauf $A$. RNAi mediated silencing of actin expression in adult Litomosoides sigmodontis is specific, persistent and results in a phenotype. Int J Parasitol. 2006:36:661-9.

18. Kamath RS, Ahringer J. Genome-wide RNAi screening in Caenorhabditis elegans. Methods. 2003;30:313-21.

19. Song C, Gallup JM, Day TA, Bartholomay LC, Kimber MJ. Development of an in vivo RNAi protocol to investigate gene function in the filarial nematode, Brugia malayi. PLoS Pathog. 2010;6:e1001239.

20. Jinkichi F, Soichi I, Toshio I, Tetsuo N, Ken T, Tadashi T, et al. Heterotopic parasitism of Setaria digitata (Linstow, 1906) in the heart of a cattle. Japanese J Vet Sci. 1985;47:999-1002.

21. Bal M, Das MK. Antigenicity of a filarial protease from Setaria digitata in Wuchereria bancrofti infection. Ann Trop Med Parasitol. 1999;93:279-88.

22. Decruse SW, Raj RK. Histological studies on female Setaria digitata (von Linstow 1906), a filaria of bovine, Bos indicus. Proc Anim Sci. 1990;99:103-12.

23. Hawking F \& World Health Organization. Diethylcarbamazine: a review of the literature with special reference to its pharmacodynamics, toxicity, and use in the therapy of onchocerciasis and other filarial infections. Geneva: World Health Organization; 1978. http://www.who.int/iris/handle/10665/70735

24. Rodrigo WWP, Dassanayake RS, Voronin D. Novel parasitic nematode-specific protein of Setaria digitata largely localized in longitudinal muscles, reproductive systems and developing embryos. Exp Parasitol. 2014;141:12-20.

25. Lendner M, Doligalska M, Lucius R, Hartmann S. Attempts to establish RNA interference in the parasitic nematode Heligmosomoides polygyrus. Mol Biochem Parasitol. 2008;161:21-31.

26. Tung KC, Cheng FP, Lai CH, Wang KS, Wang JS, Lee WM. Demonstration of vector competence of Culex quinquefasciatus (Diptera: Culicidae) for Setaria digitata. Vet Parasitol. 2004;123:279-84. 
27. Gerberg EJ. Manual for mosquito rearing and experimental techniques. Bull Am Mosq Control Assoc. 1970;5:1-109.

28. Hayes RO. Determination of a physiological saline solution for Aedes aegypti (L.). J Econ Entomol. 1953;46:624-7.

29. Benedict M, Howell P. 3.8 Mosquito Anesthesia. In: Methods in Anopheles Research. 2nd ed. Atlanta: bei RESOURCES; 2010. p. 1-4.

30. Garver L, Dimopoulos G. Protocol for RNAi assays in adult mosquitoes (A. gambiae). J Vis Exp. 2007;5:230.

31. Luna BM, Juhn J, James AA. Injection of dsRNA into female A. aegypti mosquitoes. J Vis Exp. 2007;215:224.

32. Ford L, Zhang J, Liu J, Hashmi S, Fuhrman JA, Oksov Y, et al. Functional analysis of the cathepsin-like cysteine protease genes in adult Brugia malayi using RNA interference. PLoS Negl Trop Dis. 2009;3:e377.

33. Ma HB, Lu Q, Liang J, Zhang XY. Functional analysis of the cellulase gene of the pine wood nematode, Bursaphelenchus xylophilus, using RNA interference. Genet Mol Res. 2011;10:1931-41.

34. Aboobaker AA, Blaxter ML. Use of RNA interference to investigate gene function in the human filarial nematode parasite Brugia malayi. Mol Biochem Parasitol. 2003;129:41-51.

35. Lustigman S, Zhang J, Liu J, Oksov Y, Hashmi S. RNA interference targeting cathepsin $L$ and Z-like cysteine proteases of Onchocerca volvulus confirmed their essential function during L3 molting. Mol Biochem Parasitol. 2004;138:165-70.

36. Dassanayake RS, Rodrigo WWP, Karunanayake EH, Weerasena OVDSJ, Chandrasekharan NV. A putative nuclear growth factor-like globular nematode-specific protein. Bioinformation. 2009;3:370-4.

Ready to submit your research? Choose BMC and benefit from:

- fast, convenient online submission

- thorough peer review by experienced researchers in your field

- rapid publication on acceptance

- support for research data, including large and complex data types

- gold Open Access which fosters wider collaboration and increased citations

- maximum visibility for your research: over $100 \mathrm{M}$ website views per year

At $\mathrm{BMC}$, research is always in progress.

Learn more biomedcentral.com/submissions 